\title{
Chronic myeloid leukemia: In pursuit of perfection
}

\author{
Vishal Jayakar
}

\section{Abstract}

The resounding success of imatinib (IM) as front line treatment in patients with chronic myeloid leukemia (CML) has certainly made a paradigm shift in the therapeutic algorithm of this disorder.The precise targeting of the BCR-ABL oncogene in CML has entitled it to be the poster child of translational medicine with a well-deserved Oscar ovation from the oncology community. Clinicians are now empowered with first-, second- and third-generation tyrosine kinases, as well as advanced molecular tools to monitor disease and characterize resistance. We have come a long way in successfully managing these patients, but there are still a significant few unmet clinical needs which need addressing and targeting to optimize clinical outcomes. This review focuses on 4 such pertinent and relevant clinical issues, which still need ironing out to fulfill our ambition of achieving 'perfection' in this patient cohort.

Key words: Chronic phase chronic myeloid leukemia, cure, tyrosine kinase inhibitor

\section{Introduction}

Elucidation of the pathogenesis of CML and introduction of tyrosine kinase inhibitors (TKI) has transformed this malignancy from being invariably fatal to a disorder with a chronic course expected to have a near-normal life expectancy, with preservation of an acceptable quality of life. In recent epidemiological data, the survival of younger patients treated with IM is within $10-20 \%$ of the life expectancy of age-matched Peers. ${ }^{[1]}$ In the pivotal IRIS trial, the established overall survival (OS) of patients who received IM as an initial therapy was $89 \%$ at 5 years and $85 \%$ at 8 years $(93 \%$ when deaths only related to CML were considered). ${ }^{[2]}$

With 3 TKI's available for newly-diagnosed patients with chronic phase CML (FDA approved), the landscape has become comparable to that of a chronic disease such as hypertension with a choice between different first line agents.

Consultant Haemato-Oncologist, Kingston and Royal Marsden

Hospitals, London, UK

Correspondence to: Dr.Vishal Jayakar,

E-mail: vishal.jayakar@kingstonhospital.nhs.uk

\begin{tabular}{|l|l|}
\hline \multicolumn{3}{|c|}{ Access this article online } \\
\hline Quick Response Code: & Website: \\
\hline & www.sajc.org \\
\hline & \\
\hline
\end{tabular}

However, a significant minority of patients still have an adverse outcome. Blast crisis occurs at an incidence of $1-2 \%$ per year, and once this has occurred, treatment options are limited and survival is short. A quarter of patients fail on IM therapy either because of intolerance or resistance and need alternative strategies to salvage them. ${ }^{[2]}$ The monitoring process for patients is rigorous with strict time-dependent variables, and there are still a few concerns in harmonizing the methodologies involving transcript assays. The dream of discontinuation of treatment and achieving cure still seems distant.

This article aims at addressing these issues critically by an up to date evaluation of present evidence in an attempt to provide answers to the following 4 questions

Q1. Is there a need to improve first line therapy with Imatinib and how may we achieve that?

Q2. Do we need better time-dependent goal posts?

Q3. What is the best strategy when imatinib fails?

Q4. Can we be aim for treatment discontinuation and dream of a cure?

\section{Q1: Is there a need to improve first line therapy with Imatinib and how may we achieve that?}

Imatinib is highly effective in treatment of chronic phase CML. In the seminal IRIS trial, a complete cytogenic response (CCyr-No $\mathrm{Ph}+$ ve metaphases detected) was achieved in $83 \%$ of patients, with a projected 8 -year eventfree survival of $81 \%$ and overall survival of $85 \%$. In this trial, $17 \%$ of patients never achieved CCyr, 15\% achieved CCyr but eventually lost it, and nearly 5\% were intolerant to imatinib. ${ }^{[2]}$ Thus, approximately one-third of the patients did not have a desired outcome. 
However, the true incidence of resistance to imatinib may be underestimated in this trial and may be higher than the depicted values.

Results from the Hammersmith UK data on 204 newlydiagnosed chronic phase CML patients estimated that an individual's likelihood of remaining in CCyr while receiving IM 5 years after diagnosis was approximately $63 \%{ }^{[3]}$

One universal concern surrounding clinical trials is the extra commitment of both practitioners and patients to optimal outcomes, and the fact that they may give superior results when compared to real-world experiences. Lucas et al., recently published a retrospective evaluation of 84 patients where in $51-58 \%$ would have been considered to be IM-resistant by failing to achieve $\mathrm{CCyr}$ at 18 months. ${ }^{[4]}$

These studies indicate that both primary and secondary resistance to IM remains a venerable challenge in a significant minority of newly-diagnosed chronic phase CML patients, leaving a sizeable room for an improvement.

\section{How may be improve front line therapy?}

Increasing the dose of IM?

To formally address this question, the TOPS (Tyrosine Kinase inhibitor optimization and selectivity) study randomized 476 patients $2: 1$ to IM $400 \mathrm{mg}$ or $800 \mathrm{mg}$ daily. MMR rates were higher in the $800 \mathrm{mg}$ arm at 3 and 6 months and so were the CCyr rates at 6 months. However, there was no statistically significant difference in MMR ( $46 \%$ vs. $40 \%, P=0.203)$ or CCyr $(70 \%$ vs. $66 \%$, $P=0.347)$ at 12 months. ${ }^{[5]}$ Longer follow-up is required to ascertain whether the faster responses achieved with 800 mg IM will translate in to clinical benefit.

Needless to say, the incidence of hematologic and nonhematologic toxicities was much higher in the high dose arm. 2 other studies have attempted to answer the same question, albeit with a different design.

The German CML IV study (1,014 patients), which used a tolerability-adapted IM-dose escalation scheme, found that patients who were able to tolerate $800 \mathrm{mg}$ IM had a significantly higher MMR rate at 12 months $(59 \%$ vs. $44 \%$, $P<0.01)^{[6]}$

The TIDEL I investigated higher dose IM in 103 patients with newly-diagnosed CML patients using $600 \mathrm{mg} /$ day with dose escalation to $800 \mathrm{mg}$ /day for sub-optimal response. Both EFS (89\% vs. $56 \%, P<0.01)$ and CCyr $(96 \%$ vs. $80 \%, P<0.01)$ at 60 months was significantly higher in patients taking $>600 \mathrm{mg}$ /day compared with those who had been dose reduced to $<600 \mathrm{mg} /$ day. ${ }^{[7]}$ These results suggest that patients who are able to tolerate higher doses of imatinib fare better than patients who experience an unacceptable toxicity.

There is enough evidence to suggest that IM is actively pumped in to cells by the cationic transporter HOCT1. Some studies have indicated that higher doses of IM may be more effective at achieving clinically beneficial BCR-ABL inhibition in patients with low level of HOCT1 expression. ${ }^{[8]}$ However, these measurements are not routinely available for all patients, and there are significant issues with reproducibility of HOCT1 assays from various labs.

Conclusion - Despite mounting evidence that patients who can tolerate higher doses of IM can derive clinical benefit, the greater incidence of toxicity, conflicting and not so mature data from phase 3 studies and the cost associated with higher doses of IM make it difficult to confidently suggest doses higher than $400 \mathrm{mg}$ /day as 'standard of care' in newly-diagnosed chronic phase patients.

\section{Combination of IM with IFN-a}

2 studies viz the SPIRIT and German CML- IV have investigated the ability of addition of pegylated IFN to IM to increase the achievement of CCyr and MMR over IM alone.

The SPIRIT trial had 4 arms of randomization as shown in Figure 1. The arm, which showed the maximal benefit with respect to achieving an MMR at 18 months, was the arm of IM $400 \mathrm{mg} /$ day with pegylated IFN $90 \mu \mathrm{g}$ weekly. CMR rates at 24 months were the highest in this arm as well, but this combination failed to demonstrate a significant reduction in $\mathrm{AP}$ or $\mathrm{BC}$ disease transformation and death after 48 months of follow-up. ${ }^{[9]}$

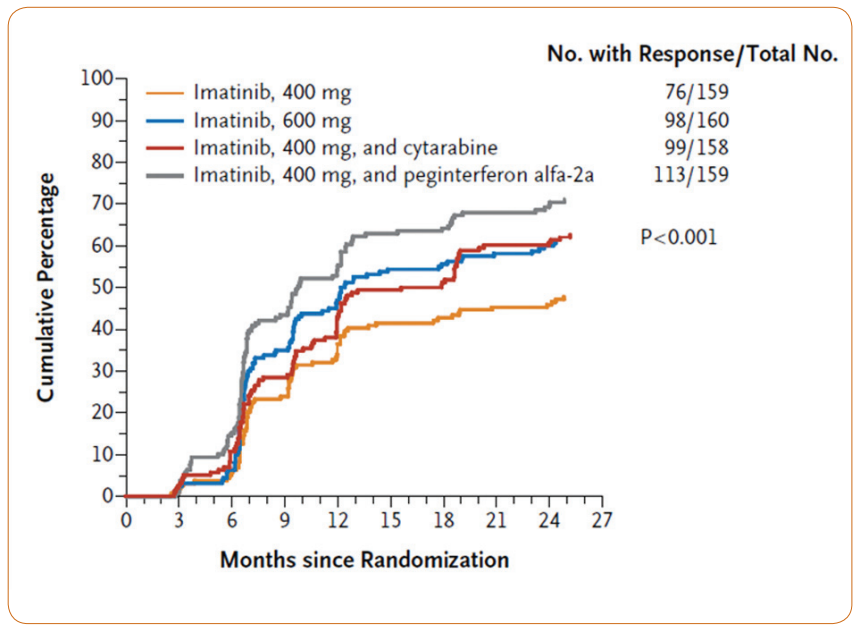

Figure 1: SPIRIT study demonstrating that the arm of imatinib 400 $\mathrm{mg} / \mathrm{d}$ with pegylated IFN $90 \mathrm{\mu g}$ weekly was superior in achieving MMR 
The German CML IV study failed to show an advantage of pegylated IFN plus IM $400 \mathrm{mg} /$ day over IM $400 \mathrm{mg}$ /day alone in the achievement of MMR at 12 months. ${ }^{[6]}$

This conflicting result may be related to different formulations of IFN used; in the SPIRIT study, pegylated IFN- $\alpha 2 \mathrm{a}$ at a dose of $90 \mu \mathrm{g}$ weekly was used, whereas the German CML IV added IFN- $\alpha 6$ weeks after the start of IM at an initial dose of 1.5 million units 3 times per week and increased the dose up to 3 million units 3 times a week, depending upon tolerability.

The second generation TKI's (as noted below) also achieve response rates superior to IM $400 \mathrm{mg} /$ day in newlydiagnosed chronic phase CML patients and are expected to be associated with less toxicity than the imatinib-IFN combination.

Conclusion - Hence, the potential benefit of IFN in combination with standard dose IM is still unclear and no strong recommendation about combination strategies can be made on basis of currently available evidence.

\section{Second generation Tyrosine Kinase inhibitors}

On the basis of the higher in vitro potency of the second generation TKI, with lesser propensity to trigger mutations and a reasonable toxicity profile as second line therapy, these agents became attractive candidates for first line treatment.

Several phase 2 studies of dasatinib and nilotinib have demonstrated high rates of CCyr and MMR when used in newly-diagnosed patients with chronic phase CML. In the single arm phase 2 study of dasatinib at M D Anderson, the CCyr and MMR figures were $82 \%$ and $98 \%$, respectively. ${ }^{[10]}$ In a similar study at the same institution, nilotinib was associated with similar results in front line setting, with a cumulative CCyr rate of $98 \%$ and a MMR rate of $76 \% .^{[11]}$

Randomized phase 3 studies have recently demonstrated the superiority of both dasatinib and nilotinib in achievement of both CCyr and MMR compared to standard dose IM in newly-diagnosed chronic phase patients.

\section{ENESTnd- Nilotinib}

In the multicenter phase 3 randomized study ENESTnd (Evaluating nilotinib efficacy and safety in clinical trialsnewly diagnosed patients), 846 patients were randomized in a 1:1:1 ratio to receive either nilotinib $300 \mathrm{mg} B D$, nilotinib $400 \mathrm{mg} \mathrm{BD}$, or IM $400 \mathrm{mg} /$ day. The $300 \mathrm{~g}$ BD dose emerged as the most superior [Figure 2]. ${ }^{[12]}$
Achievement of CCyr by 24 months was significantly higher for nilotinib $300 \mathrm{mg}$ BD compared with IM $400 \mathrm{mg}$ daily ( $87 \%$ vs. $77 \%, P=0.0018$ ). The MMR rate by 24 months remained significantly higher for nilotinib $300 \mathrm{mg}$ BD (71\%, $P<0.001)$ and nilotinib $400 \mathrm{mg}$ BD $(67 \%, P<$ 0.001 ) compared with IM $400 \mathrm{mg} /$ day (44\%).

The achievement of an MMR remained higher for both nilotinib arms across all Sokal risk groups. Interestingly, the rates of CMR (Complete molecular response, $4.5 \mathrm{log}$ reduction) were also higher for nilotinib $300 \mathrm{mg} \mathrm{BD}$ as opposed to IM $400 \mathrm{mg} /$ day (44\% vs. $20 \%, P<0.001$ ).

\section{DASISION- Dasatinib}

In the multinational Dasatinib vs. Imatinib study in treatment-naïve CML patients [Figure 3], 519 patients were randomized to receive either $100 \mathrm{mg}$ dasatinib daily or IM $400 \mathrm{mg}$ daily. ${ }^{[13]}$

At 24 months, the rates of MMR were significantly higher for dasatinib $(64 \%$ vs. $46 \%, P<0.001)$ as compared to standard dose IM. CCyr rates at 18 months were higher for

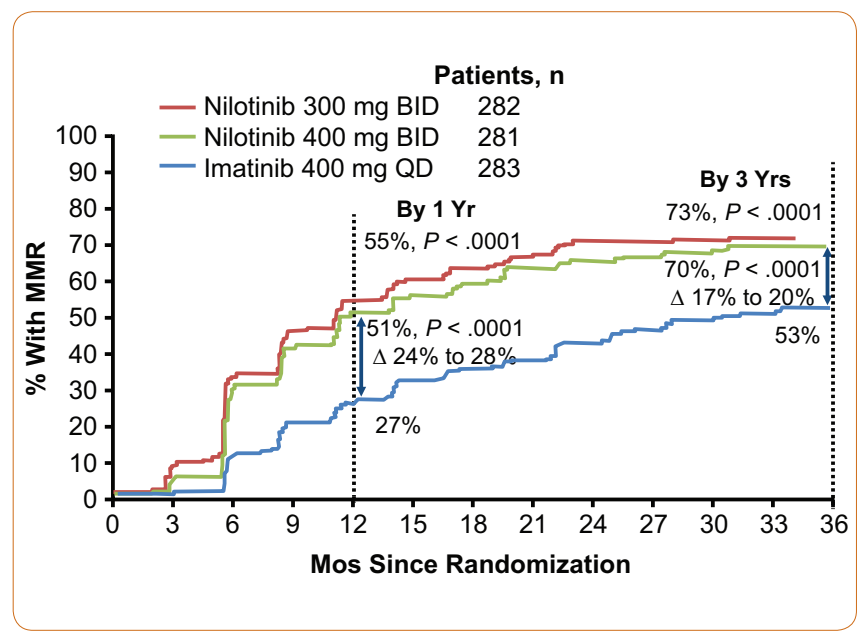

Figure 2: ENESTnd study demonstrating superior MMR figures at 24 months with Nilotinib $300 \mathrm{mg}$ BD compared to Imatinib $400 \mathrm{mg}$ daily

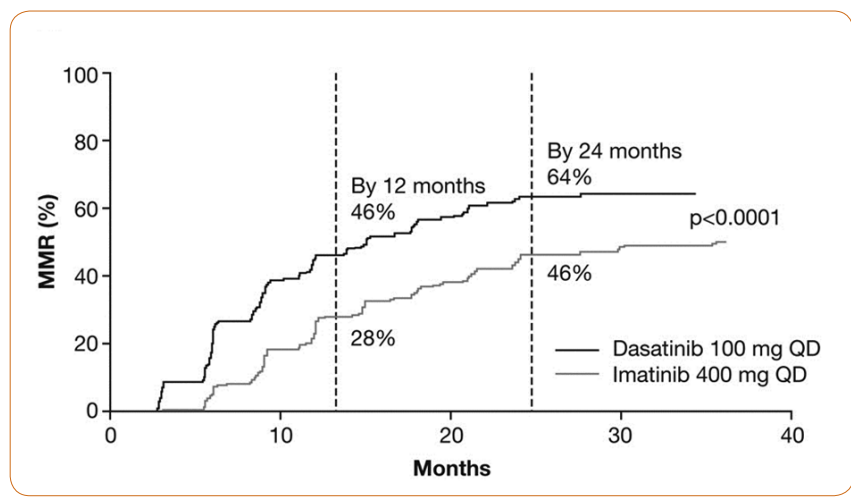

Figure 3: DASISION study showing superior results for Dasatinib $100 \mathrm{mg}$ daily achieving MMR at 24 months in comparison to Imatinib $400 \mathrm{mg}$ daily 
dasatinib ( $78 \%$ vs. $70 \%, P=0.037)$.

In a phase 3 multinational randomized BELA trial (Bosutinib efficacy and safety in newly diagnosed CML), 502 patients with newly-diagnosed chronic phase CML patients were randomized to bosutinib $500 \mathrm{mg}$ /day vs. IM $400 \mathrm{mg} /$ day. Unfortunately, the trial failed to achieve its primary end point because of lack of difference in the 12 month CCyr rates between the arms $(70 \%$ vs. $68 \%, P$ $=0.6$ ). There were higher rates of discontinuation in the bosutinib arm, primarily due to GI toxicity. ${ }^{[14]}$

The results of the DASISION and ENESTnd were encouraging enough for both dasatinib and nilotinib to get FDA approval for first line usage in chronic phase CML alongside IM, but whether dasatinib and nilotinib will have an OS survival benefit in patients with CML needs to be ascertained in the coming years as this data continues to mature.

Clearly the most obvious benefit with both these TKI is the earlier achievement of CCyr and MMR. It is tempting to speculate that earlier achievement of these milestones in patients with chronic phase CML will translate in to better EFS and OS since there is data to support that the longer it takes to achieve a $\mathrm{CCyr}$, the higher the probability of experiencing disease progression.

Intriguingly, both the DASISION and ENESTnd trials have shown that fewer patients in the nilotinib and dasatinib arms progressed to AP and/or BC as compared to standard dose IM within the first 24 months [Figure 4].

This certainly provides strong evidence for advocation of the notion that achieving CCyr and MMR earlier in the temporal profile is of clinical benefit to the patients by minimizing risk of disease progression.

Conclusion - Given the historic prognostic significance of cytogenetic and molecular responses, there is a substantial

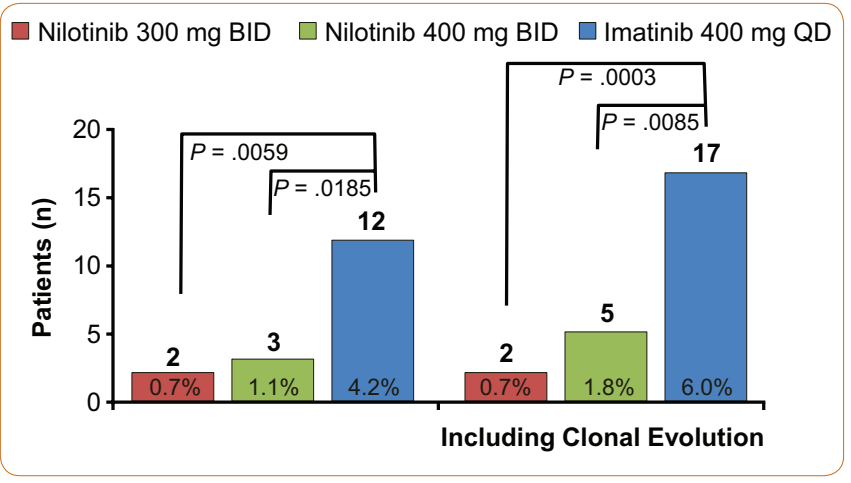

Figure 4: ENESTnd results demonstrating lesser patients progressing to accelerated phase or blast crisis with Nilotinib $300 \mathrm{mg} \mathrm{BD}$ compared to Imatinib $\mathbf{4 0 0} \mathbf{~ m g}$ daily optimism that the second generation-TKI will convincingly improve longer-term outcomes relative to IM, but longer follow-up on both these studies are required to definitively stamp this issue.

\section{Allogenic stem cell transplantation}

Allogenic stem cell transplantation (SCT) was frontline therapy among eligible patients with CML prior to the introduction of IM in 1999.

In the IM era, its role receded to second line therapy reserved for patients with IM failure. With the wide usage of new second and third generation TKI, which effectively salvage at least $50 \%$ of IM resistant patients, its position in the CML armamentarium is increasingly considered third line salvage for patients who fail IM and second generation TKI.

However, the following clinical situations might prompt consideration of allogenic SCT as first line therapy

- Patients with p190 chronic phase CML

- Identical twin sibling

- In developing countries where the cost of transplant as a fixed-cost curative option is much less than the economic burden of daily IM indefinitely (However, this may change with the availability of generic IM in 2015)

- Patients with T315I mutations who are resistant to all 3 FDA-approved TKI. Ponatinib, a third generation TKI, has shown promising results in this patient cohort and should be used as bridging therapy to the SCT. ${ }^{[15]}$

\section{Q2: Do we need better time dependent goal posts?}

The 3 contentious issues in this domain are

- Clarity on 'sub-optimal' responses and treatment algorithms for patients who fall in this category

- Inter laboratory variations in BCR-ABL measurements

- Milestones for patients on second generation TKI

- Can we have a simpler goal post?

We have clear guidelines by the ELN regarding monitoring

Table 1: Investigations recommended by European Leukemia Net for patients with chronic phase CML patients

\begin{tabular}{ll}
\hline At diagnosis & At follow up \\
\hline BM Asipration cytology & BM cytogenetics 3 monthly until \\
(+ Biopsy) & CCyR \\
BM Cytogenetics & qRT-PCR 3 to 6 monthly \\
PM Multiplex PCR & (Interphase FISP to confirm CRyR \\
(for type of bcr-abl transcript) & if PCR is not available) \\
(PB FISH) & Mutation analysis in cases with \\
& - Lack/loss of MMoIR \\
& $-5 \times$ increase in bcr-abl transcript \\
\hline
\end{tabular}


patients with newly-diagnosed chronic phase CML [Table 1]. ${ }^{[16]}$

CCyr seems to be most reproducible and reliable surrogate end point for long term prognosis in these patients. In the IRIS study, patients who achieved a MMR by 18 months had a $100 \%$ freedom from progression to $\mathrm{AP} / \mathrm{BC}$ and $95 \%$ EFS at 7 years ${ }^{[17]}$ making this another goal post to be achieved in the ELN and NCCN recommendations. The degree to which achievement of MMR adds clinical benefit to the achievement of CCyr is presently unclear.

Nevertheless, MMR is an important landmark with proven prognostic significance. However, wide variations in the methods used to quantify BCR-ABL and the lack of widely-accepted standards have led to considerable variations in results making comparability between different laboratories difficult. An international program is now underway to harmonize the reporting of results according to an international scale. Laboratory-specific conversion factors are only valid for particular instruments and particular standard operating procedures; any change in laboratory protocols or upgrade of equipment will necessitate recalculation of the conversion factor. ${ }^{[18]}$

Resistance is neatly defined by these guidelines and certainly warrants change of treatment other than IM.

However, the question of when to change IM therapy has been muddied by the proposal of definitions of sub-optimal and optimal responses.

Unlike failure, a sub-optimal response is a status which puts clinicians ill at ease, but which may or may not predict for IM resistance or worse long-term prognosis.

According to the ELN, the management for this patient cohort includes continuation of therapy with more rigorous monitoring, use of high-dose IM or changing to a second

Table 2: ELN guidelines for failure and sub optimal responses in patients with $\mathrm{CML}$

\begin{tabular}{llll}
\hline Time & Failure & $\begin{array}{l}\text { Suboptimal } \\
\text { response }\end{array}$ & Warnings \\
\hline Diagnosis & - & - & $\begin{array}{l}\text { High risk CCA } \\
\text { in Ph1+cells }\end{array}$ \\
3 months & No CHR & No CyR & \\
6 months & $<$ CHR, no CyR & $<$ PCyR & \\
12 months & $<$ PCyR & $<$ CCyR & $<$ MMoIR \\
18 months & $<$ CCyR & $<$ MMoIR & \\
Anytime & CCA in Ph+cells, & $\begin{array}{l}\text { Loss of } \\
\text { MmoIR, IM }\end{array}$ & $\begin{array}{l}\text { Any increase in } \\
\text { bcr-abI transcript } \\
\end{array}$ \\
& $\begin{array}{l}\text { loss of CHR, } \\
\text { loss of CCyR, }\end{array}$ & $\begin{array}{l}\text { sensitive } \\
\text { mutations }\end{array}$ & Ph1-cells \\
& $\begin{array}{l}\text { IM insensititive } \\
\text { mutations }\end{array}$ & & \\
\hline
\end{tabular}

generation-TKI. This has led to the need of heterogeneous treatment algorithms in patients with sub-optimal responses amongst physicians [Table 2]. ${ }^{[19]}$

Another issue of important concern is treatment milestones on second generation TKI used, either as front line or in the second line setting. Given that the cytogenetic and molecular responses are rapidly achieved with these agents, evolving data suggest that a failure to achieve a major cytogenetic response $(<35 \% \mathrm{Ph}+$ metaphases $)$ within 12 months of an initiation portends a poor prognosis. ${ }^{[20]}$ It is, therefore, reasonable to consider allogenic SCT in this cohort of patients but again, we need more prospective and mature data to confidently support this.

The current monitoring process is rigorous, and attempts have been made to identify a reliable and robust early marker to predict whether a patient would do well on IM earlier on. Hughes et al., suggested that patients who achieve only a 1-log reduction (approximately equivalent to a BCR-ABL: $\mathrm{ABL}$ ratio of $10 \%$ in the international scale) at 3 months have a significantly lower probability of achieving a MMR (13\% at 30 months) than those with 1to 2- $\log$ reduction $(69 \%)$ or those with a more than 2-log reduction (100\%). ${ }^{[21]}$ More recently, Quintas-Cardama et al., reported similar results. ${ }^{[22]}$

Marin et al., from Hammersmith, UK, have elegantly confirmed this data in a recent publication in JCO. ${ }^{[23]} \mathrm{By}$ using a receiver operating characteristic curve to discover the optimal cut-off in transcript levels, they determined that a value of less than $9.84 \%$ at 3 months identifies those patients with the best outcome. This level of response at 3 months was independently associated with OS, PFS, EFS, and current CCyr survival (c-CCyr). ${ }^{[23]}$ Optimally, then we would aim for a transcript level of $<10 \%$ at 3 months from start of therapy. Should patients who fail to achieve this landmark at 3 months change treatment from standard dose IM and move on to second generation TKI?

Prospective studies are needed to answer this confidently, but the study by Marin et al., has offered us some meaningful insight.

It is likely that in the future, all we need to ascertain to change first line treatment is a failure to achieve a $>1-\log$ reduction of $\mathrm{BCR}-\mathrm{ABL}$ transcripts at 3 months.

\section{Q3: What is the best strategy when imatinib fails?} Imatinib failure should be looked at under the following 3 headings [Figure 5];

A patient has TKI intolerance if one or more of the following criteria have been met:

1) Any life threatening grade 4 non-hematological toxicity

2) Any grade $3 / 4$ non-hematological toxicity that has 
recurred despite dose reductions

3) Any grade 2 non-hematological toxicity that persists for more than a month despite optimal supportive measures

4) Grade 3-4 hematological toxicity that is unresponsive to supportive measures and would require dose reductions below the accepted minimal effective dose.

Beyond these clear-cut definitions of intolerance, there is a gray area governed as much by objective findings as by the availability of alternatives that may offer a better quality of life. ${ }^{[24]}$ It has been our experience that quite a few patients who have been on IM for more than 3 years experience profound fatigue significantly affecting quality of life, which does reverse on stopping IM and switching to a second generation TKI. We and others do slot this as intolerance to long term IM and recommend treatment change.

Non-adherence to IM is a much bigger problem than initially conceived.

In a study performed at Hammersmith London, 87 patients with chronic phase CML treated with 400 mg IM for a median of 59.7 months had adherence monitored during a 3-month period within CCyr using a microelectronic monitoring device. $26.4 \%$ had adherence of $<90 \%$ and $14 \%$ had adherence of $<80 \%$. There was a strong correlation between adherence rate and the 6-year probability of achieving an MMR (28.4\% vs. 94.5\%) and CMR ( $0 \%$ vs. $43.8 \%$ ). Multivariate analysis identified adherence and OCT-1 levels as the only independent predictors of MMR. ${ }^{[25]}$ Further, poor adherence is the principal factor contributing to the loss of cytogenetic responses and treatment failures in patients on long term therapy.

Hence, patients with secondary resistance should be questioned thoroughly for compliance. Drug-delivery devices with reminder function, diaries, or text-messaging reminders may help to increase an adherence. Physicians and pharmacists have an important role in patient education to improve 'Imatinib truancy' since improving an adherence

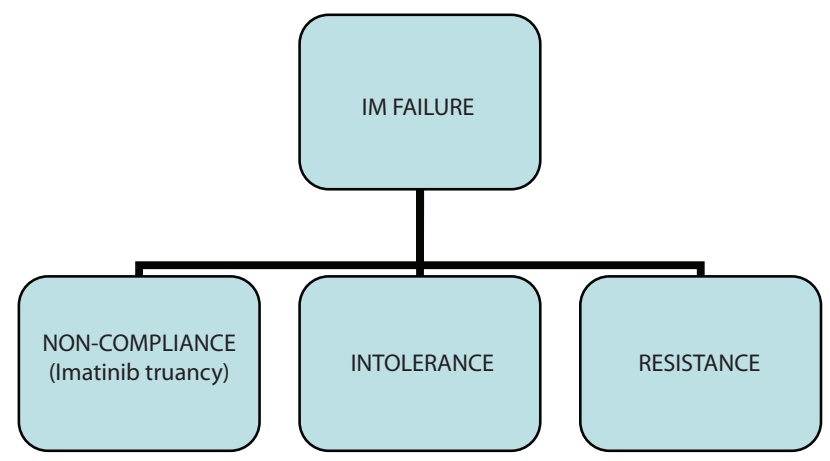

Figure 5: Causes of imatinib failure may not only optimize clinical outcomes, but may also reduce the economic burden of CML. ${ }^{[26]}$

Molecular mechanisms responsible for true primary and secondary resistance to IM have been the subject of an intense research. Many cases involve escape of BCRABL inhibition, either through kinase domain mutations or presumed overproduction of BCR-ABL via genomic amplification or through acquisition of additional $\mathrm{Ph}$ chromosomes in the resistant clone. A small minority have resistance mechanisms independent of BCR-ABL. A complete elucidation of mechanisms of TKI resistance is beyond the scope of this review.

Once a patient fails primary TKI therapy, one cannot be complacent that a second generation TKI can singularly save the day. Alarm bells must start ringing and contemplation of an allogenic SCT should be made, especially if the patient has transited from chronic phase to AP or worse BC. Irrespective of the mechanism of resistance, allogenic SCT should be strongly considered in patients with $\mathrm{AP} / \mathrm{BC}$, if feasible (age, performance status, donor availability). Second generation TKI can be used as bridging treatment to transplant procedure.

In patients with true resistance in chronic phase, second generation TKI cause a CCyr in $50 \%$ of cases with durable responses at the end of 2 years. ${ }^{[27]}$ The remaining half of patients need to be considered for allogenic SCT or a clinical trial, and second generation TKI salvage can be perceived as a bridging therapy to these robust modalities. A treatment algorithm for resistant patients is charted in Figure 6 .

The choice of which approved second generation TKI (dasatinib vs. nilotinib) therapy to proceed with in case of IM failure would rest on the following in this particular order

Mutational analysis

Patient co-morbidities

Physician preference and familiarity

The T3151 mutation, which imposes a bulky isoleucine residue in the BCR-ABL ATP pocket making it inaccessible to all 3 TKI (IM, dastinib, nilotinib), was considered as an unbeatable mutation with allogenic SCT as the only treatment option. ${ }^{[28]}$ However, encouraging results from a third generation TKI ponatinib have heightened hopes amongst hemato-oncologists of using this new agent as salvage and/or bridging therapy. Impressively, of 9 patients with chronic phase CML and the T3151 mutation at study entry, 8 achieved CCyr. ${ }^{[15]}$ To date, these responses have been durable up to 15 months. Ponatinib is currently being investigated in a phase 2 clinical trial in all phases of $\mathrm{CML}$ and $\mathrm{Ph}+\mathrm{ve} \mathrm{ALL}$ with resistance to either dasatinib or nilotinib. 


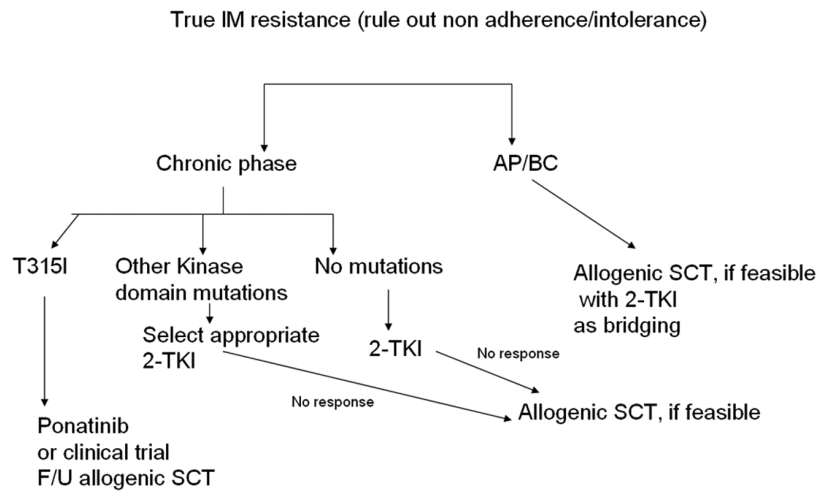

Figure 6: Clinical algorithm for patients with chronic phase CML presenting with true imatinib resistance

If Ponatinib is well-tolerated, assessing its response rate and durability in treatment-naïve chronic phase CML and $\mathrm{Ph}+\mathrm{ve}$ patients will be of great interest.

DCC-2036, an allosteric inhibitor of BCR-ABL and omacetaxine, are being investigated in phase $1 / 2$ studies for similar purposes. ${ }^{[29]}$

If the patient has mutation in the kinase domain other than T315I, the nature of the mutation would determine the choice between dasatinib vs. nilotinib. For example, the F317L and V299L mutations are resistant to dasatinib making the clinician inclined towards commencing nilotinib in these patients. Alternatively, the nilotinib poorrisk $\mathrm{E} 255 \mathrm{~K} / \mathrm{V}, \mathrm{F} 359 \mathrm{C} / \mathrm{V}$, and $\mathrm{Y} 253 \mathrm{H}$ mutations appear sensitive to dasatinib. ${ }^{[30,31]}$

The toxicity profile and the co-morbidities of the patient might influence the decision of the second generation TKI as well. For example, patients with lung pathologies or congestive cardiac failure who are likely to develop pleural effusions could avoid dasatinib because of higher propensity of pleural effusions seen with dasatinib treatment. Likewise, a clinician might be less inclined in switching to nilotinib in patients with previous pancreatitis or diabetes because of the side effect profile of nilotinib.

Nilotinib needs twice-daily dosing, whereas dasatinib is a single-daily dose which might influence the choice of drug according to patient preference. Physician familiarity might be another contributing factor in making this choice.

\section{Q4: Can we be aim for treatment discontinuation and} dream of a cure?

Among the most intriguing clinical questions remaining in the management of CML is whether patients could eventually discontinue treatment and be cured.
The current recommendation is to continue TKI therapy indefinitely. Several groups are assessing the possibility of stopping imatinib after achieving CMR (complete molecular response with undetectable $\mathrm{BCR}-\mathrm{ABL}$ transcripts generally amounting to a $4.5-5 \log$ reduction).

The French STIM study, the most mature of its kind, prospectively assessed IM discontinuation in $100 \mathrm{CML}$ patients in CMR of more than 2 years duration. In the interim analysis, $61 \%$ patients relapsed with $98 \%$ of relapses occurring in the first 7 months. The majority of relapses occurred only at a molecular level, and resumption of IM rapidly restored CMR. The only patient characteristic that proved useful as a predictor of relapse risk was Sokal score: The probability of stable CMR was $54 \%$ in the low risk group vs. $13 \%$ in patients with a high risk score. ${ }^{[32]}$

The Australian Leukemia and Lymphoma group (ALLG) CML8 study of IM cessation in CMR has shown identical results in a smaller group of patients (D.M. Ross and T.P. Hughes, personal communication to ASH).

A pan-European clinical study is underway by the ELN to address this.

There have been no studies so far of therapy withdrawal in patients receiving newer TKI.

The reason why the remaining $39 \%$ of patients in the STIM study continued to be in CMR at a median follow-up of 17 months is the subject of an intense research, and the reader is referred to the review article by J. Melo and D. Ross published in the education book of ASH-2011. ${ }^{[33]}$

However, for our patients to be eligible to be considered to discontinue IM (the first step towards cure), they have to achieve CMR. Only $32 \%$ of patients treated with IM achieved sustained CMR after a median follow-up of 70 months. ${ }^{[34]}$ Our current efforts should be geared towards increasing this number so that the possibility of discontinuation can be offered to a larger number of patients.

\section{Conclusion}

Though huge strides have been made in the CML domain, oncologists still face significant problems with patient monitoring and IM resistance. IM has changed this malignancy in to a chronic disease with very few patients progressing to advanced phase. Though an 'operational cure' has been achieved by IM in a majority of CML patients, the dream of 'true cure' with IM discontinuation is still distant. We remain optimistic that the coming decade will certainly see many more basic 
and translational research advances making the vision of cure in the truest sense possible for the CML patient clientele.

\section{Acknowledgment}

I am grateful to Dr Helen Sykes (Head of HematologyKingston Hospital) for critical evaluation of the manuscript.

\section{References}

1. Björkholm M, Ohm L, Eloranta S, Derolf A, Hultcrantz M, Sjöberg J, et al. Success story of targeted therapy in chronic myeloid leukemia: A population based study of patients diagnosed in Sweden from 1973 to 2008. J Clin Oncol 2011;29:2514-20.

2. Michael Deininger, Stephen G O'Brien, François Guilhot, John M Goldman, Andreas Hochhaus, Timothy P. Hughes et al. IRIS 8-year follow up: Sustained survival and low risk for progression of events in patients with newly diagnosed CML treated with imatinib. Blood 2009;114:abstract 1126.

3. de Lavallade H, Apperley JF, Khorashad JS, Milojkovic D, Reid $A G$, Bua $M$, et al. Imatinib for newly diagnosed patients with CML: Incidence of sustained responses in an intention-to-treat analysis. J Clin Oncol 2008;26:3358-63.

4. Lucas CM, Wang L, Austin GM, Knight K, Watmough SJ, Shwe $\mathrm{KH}$, et al. A population study of imatinib in CML demonstrates lower efficacy than in clinical trials. Leukemia 2008;22: 1963-6.

5. Cortes JE, Baccarani M, Guilhot F, Druker BJ, Branford S, Kim DW, et al. Phase 3 randomized open-label study of daily imatinib 400 $\mathrm{mg}$ versus $800 \mathrm{mg}$ in patients with newly diagnosed, previously untreated CML in chronic phase using molecular end points: Tyrosine kinase inhibition optimisation and selectivity study. J Clin Oncol 2010;28:424-30.

6. Hehlmann R, Lauseker M, Jung-Munkwitz S, Leitner A, Müller $\mathrm{MC}$, Pletsch $\mathrm{N}$, et al. Tolerability-adapted imatinib $800 \mathrm{mg} / \mathrm{d}$ versus $400 \mathrm{mg} / \mathrm{d}$ versus $400 \mathrm{mg} / \mathrm{d}$ plus interferon-alpha in newly diagnosed CML. J Clin Oncol 2011;29:1634-42.

7. Jorge E. Cortes, Michele Baccarani, François Guilhot, Brian J. Druker, Susan Branford, Dong-Wook Kim. Phase III, Randomized, open-label study of daily imatinib mesylate $400 \mathrm{mg}$ versus 800 $\mathrm{mg}$ in patients with newly diagnosed, previously untreated chronic myeloid leukemia in chronic phase using molecular end points: Tyrosine kinase inhibitor optimization and selectivity study. JCO 2010: 28;424-30.

8. Wang L, Giannoudis A, Lane S, Williamson P, Pirmohamed M, Clark RE. Expression of the uptake drug transporter hOCT 1 is an important clinical determinant of response to imatinib in CML. Clin Pharmacol Ther 2008;83:258-64.

9. Preudhomme C, Guilhot J, Nicolini FE, Guerci-Bresler A, RigalHuguet F, Maloisel F, et al. Imatinib plus peginterferon alfa-2a in CML. N Engl J Med 2010;363:2511-21.

10. Jorge E. Cortes, Dan Jones, Susan O'Brien, Elias Jabbour, Farhad Ravandi, Charles Kolle et al. Results of dasatinib therapy in patients with early chronic phase CML. J Clin Oncol 2010;28:398404.

11. Cortes JE, Jones D, O'Brien S, Jabbour E, Konopleva M, Ferrajoli $A$, et al. Nilotinib as front-line treatment for patients with CML in early chronic phase. J Clin Oncol 2010;28:392-7.

12. Saglio G, Kim DW, Issaragrisil S, le Coutre P, Etienne G, Lobo C, et al. Nilotinib versus Imatinib for newly diagnosed CML. N Engl J Med 2010;362:2251-9.

13. Kantarjian H, Shah NP, Hochhaus A, Cortes J, Shah S, Ayala M, et al. Dasatinib versus Imatinib in newly diagnosed chronic phase CML. N Engl J Med 2010;362:2260-70.

14. Gambacorti-Passerini C, Kim DW, Kantarjian HM, Brummendorf TH, Dyagil I, Griskevicius L. An ongoing phase 3 study of Bosutinib versus Imatinib in patients with newly diagnosed chronic phase CML. Blood 2010;116:208.
15. Joseph M. Gozgit, Matthew J. Wong, Lauren Moran, Scott Wardwell, Qurish K. Mohemmad, Narayana I. Narasimhan et al. Ponatinib (AP24534), a multitargeted Pan-FGFR inhibitor with activity in multiple FGFR-Amplified or mutated cancer models. Mol cancer ther; 2012: 11; 690-9.

16. Baccarani M, Cortes J, Pane F, Niederwieser D, Saglio G, Apperley J, et al. CML: An update of concepts and management recommendations of European Leukaemia Net. J Clin Oncol 2009;27:60941-51.

17. Hughes TP, Hochhaus A, Branford S, Müller MC, Kaeda JS, Foroni $\mathrm{L}$, et al. Long term prognostic significance of early molecular response to imatinib in newly diagnosed CML: An analysis from the IRIS. Blood 2010;116:3758-65.

18. Müller MC, Cross NC, Erben P, Schenk T, Hanfstein B, Ernst T, et al. Harmonization of molecular monitoring of CML therapy in Europe. Leukemia 2009;23:1957-63.

19. Kantarjian H, Cortes J. Imatinib resistant CML: Definitions and management. Vol. 5. EHA Education Book; 2011. p. 127-31.

20. Smith CC, Shah NP. TKI therapy for CML: Approach to patients with treatment naïve or refractory chronic phase disease. Hematology Am Soc Hematol Educ Program. 2011;2011:121-7.

21. Hughes $T$, Branford S. Molecular monitoring of BCR-ABL as a guide to clinical management in CML. Blood Rev 2006;20:29-41.

22. Quintás-Cardama A, Kantarjian H, Jones D, Shan J, Borthakur G, Thomas D, et al. Delayed achievement of cytogenic and molecular response is associated with increased risk of progression among patients with $\mathrm{CML}$ in early chronic phase receiving higher dose or standard dose imatinib therapy. Blood 2009;113:6315-21.

23. Marin D, Ibrahim AR, Lucas C, Gerrard G, Wang L, Szydlo RM, et al. Assessment of BCR-ABL transcript levels at 3 months is the only requirement for predicting outcome for patients with $\mathrm{CML}$ treated with tyrosine kinase inhibitors. J Clin Oncol 2012;30:232-8.

24. Jabbour E, Deninger M, Hochhaus A. Management of adverse events associated with TKI in the treatment of CML. Leukemia 2011;25:201-10.

25. Marin D, Bazeos A, Mahon FX, Eliasson L, Milojkovic D, Bua M, et al. Adherence is the critical factor for achieving molecular responses in patients with $\mathrm{CML}$ who achieve complete cytogenic responses to imatinib. J Clin Oncol 2010;28:2381-8.

26. Hochhuas A. Education session: Managing CML as a chronic disease. Hematology Am Soc Hematol Educ Program 2011;2011:128-35

27. Raditch JP. CML 2010: Where are we now and where can we go? Hematology Am Soc Hematol Educ Program 2010;2010:122-8.

28. Gorre ME, Mohammed M, Ellwood K, Hsu N, Paquette R, Rao PN, et al. Clinical resistance to STI-571 cancer therapy caused by BCR-ABL gene mutation or amplification. Science 2001;293:87680.

29. Chan WW, Wise SC, Kaufman MD, Ahn YM, Ensinger CL, Haack T, et al. Conformational control inhibition of the BCR-ABL tyrosine kinase, including the gatekeeper T315I mutant, by the switchcontrol inhibitor DCC-2036. Cancer Cell 2011;19:556-68.

30. Hughes T, Saglio G, Branford S, Soverini S, Kim DW, Müller $M C$, et al. Impact of baseline BCR-ABL mutations in response to nilotinib in patients with CML in chronic phase. J Clin Oncol 2009;27:4204-10.

31. Müller MC, Cortes JE, Kim DW, Druker BJ, Erben P, Pasquini R, et al. Dasatinib treatment of chronic phase CML: Analysis of responses according to pre-existing BCR-ABL mutations. Blood 2009; 114:4944-53.

32. Mahon FX, Réa D, Guilhot J, Guilhot F, Huguet F, Nicolini F, et al. Discontinuation of imatinib in patients with $C M L$ who have maintained complete molecular remission for at least 2 years: The prospective multicentre Stop Imatinib (STIM) trial. Lancet Oncol 2010;11:1029-35.

33. Melo JV, Ross DM. Minimal residual disease and discontinuation of therapy in CML: Can we aim at cure? Hematology Am Soc Hematol Educ Program 2011;2011:136-42.

34. Long-term molecular responses to imatinib in patients with chronic myeloid leukemia: comparison between complete 
cytogenetic responders treated in early and in late chronic phase. Palandri F, lacobucci I, Quarantelli F, Castagnetti F, Cilloni D, Baccarani M; GIMEMA Working Party on CML. Haematologica. 2007 Nov;92:1579-80.

How to site this article: Jayakar V. Chronic myeloid leukemia: In pursuit of perfection. South Asian J Cancer 2012;1:16-24.

Source of Support: Nill. Conflict of Interest: None declared.

ICON ASCO Cancer Research Workshop

$14^{\text {th }}$ and $15^{\text {th }}$ September 2012

Nashik, Maharashtra, India

Program Director: Dr Shailesh Bondarde

Email: shaileshbondarde@yahoo.com

ICON Office: khurshid.mistry@oncologyindia.org

This is a specially designed workshop for all oncology healthcare professionals who want to do clinical trials. The international faculty shall teach you how to convert a research concept or question into a full clinical trial protocol that can be implemented successfully. Newly qualified oncologists, postgraduate students and nurses are especially encouraged to attend.

This workshop is being organized by ICON Trust and ASCO. It is also supported by NCI, USA and Oncology Nursing Society, USA.

Applications should include the research concept / question/ hypothesis that you intend to study. Since there are limited seats, the applications will be screened by a special committee.

Save the Dates

$27^{\text {th }}$ ICON Meeting

$14^{\text {th }}$ to $16^{\text {th }}$ September 2012

Nashik, Maharashtra, India

Organizing Secretary: Dr Shailesh Bondarde

Email: shaileshbondarde@yahoo.com

ICON Office: khurshid.mistry@oncologyindia.org 